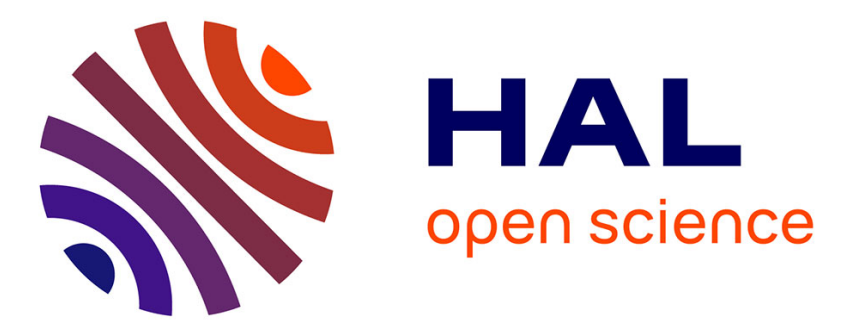

\title{
Without quality presence-absence data, discrimination metrics such as TSS can be misleading measures of model performance
}

Boris Leroy, Robin Delsol, Bernard Hugueny, Christine Meynard, Cheima Barhoumi, Morgane Barbet-Massin, Céline Bellard

\section{To cite this version:}

Boris Leroy, Robin Delsol, Bernard Hugueny, Christine Meynard, Cheima Barhoumi, et al.. Without quality presence-absence data, discrimination metrics such as TSS can be misleading measures of model performance. Journal of Biogeography, 2018, 45 (9), pp.1994-2002. 10.1111/jbi.13402 . hal02628656

\section{HAL Id: hal-02628656 \\ https://hal.inrae.fr/hal-02628656}

Submitted on 4 Dec 2020

HAL is a multi-disciplinary open access archive for the deposit and dissemination of scientific research documents, whether they are published or not. The documents may come from teaching and research institutions in France or abroad, or from public or private research centers.
L'archive ouverte pluridisciplinaire HAL, est destinée au dépôt et à la diffusion de documents scientifiques de niveau recherche, publiés ou non, émanant des établissements d'enseignement et de recherche français ou étrangers, des laboratoires publics ou privés. 


\section{Without quality presence-absence data, discrimination metrics such as TSS can be misleading measures of model performance}

\begin{abstract}
The discriminating capacity (i.e. ability to correctly classify presences and absences) of species distribution models (SDMs) is commonly evaluated with metrics such as the area under the receiving operating characteristic curve (AUC), the Kappa statistic and the true skill statistic (TSS). AUC and Kappa have been repeatedly criticized, but TSS has fared relatively well since its introduction, mainly because it has been considered as independent of prevalence. In addition, discrimination metrics have been contested because they should be calculated on presence-absence data, but are often used on presenceonly or presence-background data. Here, we investigate TSS and an alternative set of metrics-similarity indices, also known as F-measures. We first show that even in ideal conditions (i.e. perfectly random presence-absence sampling), TSS can be misleading because of its dependence on prevalence, whereas similarity/F-measures provide adequate estimations of model discrimination capacity. Second, we show that in real-world situations where sample prevalence is different from true species prevalence (i.e. biased sampling or presencepseudoabsence), no discrimination capacity metric provides adequate estimation of model discrimination capacity, including metrics specifically designed for modelling with presence-pseudoabsence data. Our conclusions are twofold. First, they unequivocally impel SDM users to understand the potential shortcomings of discrimination metrics when quality presence-absence data are lacking, and we recommend obtaining such data. Second, in the specific case of virtual species, which are increasingly used to develop and test SDM methodologies, we strongly recommend the use of similarity/F-measures, which were not biased by prevalence, contrary to TSS.
\end{abstract}

\section{1 | INTRODUCTION}

During the last decades, species distribution models (SDMs) have become one of the most commonly used tools to investigate the effects of global changes on biodiversity. Specifically, SDMs are widely used to explore the potential effects of climate change on the distribution of species of concern (Gallon et al., 2014), to anticipate the spread of invasive species (Bellard et al., 2013), and also to prioritize sites for biodiversity conservation (Leroy et al., 2014). Therefore, conservation managers increasingly rely on SDMs to implement conservation strategies and policies to mitigate the effects of climate change on biodiversity (Guisan et al., 2013). There are various methodological choices involved in the application of SDMs (e.g. data type and processing, variables, resolution, algorithms, protocols, global climate models, greenhouse gas emission scenarios), which make them particularly difficult to interpret, compare and assess. However, evaluation of their predictive accuracy is probably a common step to most SDM studies across methodological and technical choices. This evaluation allows us to quantify model performance in terms of how well predictions match observations, which is a fundamental and objective part of any theoretical, applied or methodological study.

To evaluate model predictive performance, the occurrence dataset is often partitioned into two subsets (one for calibrating models, and one for testing) and predictions are assessed in terms of whether or not they fit observations using various accuracy metrics (Araújo, Pearson, Thuiller, \& Erhard, 2005), a method called crossvalidation. Other approaches include calibrating on the full dataset and testing on an independent dataset, or, when the modelled species is a virtual, in silico, species (e.g. for testing methodological aspects), directly comparing the predicted distribution with the known true distribution (Leroy, Meynard, Bellard, \& Courchamp, 2015). Accuracy metrics can be divided into two groups: discrimination versus reliability metrics (Liu, White, \& Newell, 2009; Pearce, Pearce, Ferrier, \& Ferrier, 2000). Discrimination metrics measure classification rates, i.e. the capacity of SDMs to distinguish correctly between presence and absence sites. Reliability metrics measure whether the predicted probability is an accurate estimate of the likelihood of occurrence of the species at a given site. Here, we focus on discrimination metrics, since they are often used in the SDM literature to test model robustness; however, we stress the importance of evaluating reliability (see Meynard \& Kaplan, 2012 as well as Liu et al., 2009), for example with the Boyce index which is probably the most appropriate reliability metric for presence-only data (Boyce, Vernier, Nielsen, \& Schmiegelow, 2002; Di Cola et al., 2016; Hirzel, Randin, \& Guisan, 2006).

Discrimination metrics rely on the confusion matrix, i.e. a matrix comparing predicted versus observed presences and absences (Table 1). Such discrimination metrics have largely been borrowed from other fields of science, such as medicine and weather forecasting, rather than being specifically developed for SDM studies (Liu et al., 2009). Three classification metrics stand out in the SDMs literature: Cohen's Kappa, the area under the receiver operating characteristic curve (AUC) and the true skill statistic (TSS). AUC was 
introduced in ecology by Fielding and Bell (1997) $(2,821$ citations on Web of Science in June 2017), but has since repeatedly been criticized (Jiménez-Valverde, 2012; Lobo, Jiménez-Valverde, \& Hortal, 2010; Lobo, Jiménez-Valverde, \& Real, 2008) because of its dependence on prevalence (i.e. the proportion of recorded sites where the species is present) makes it frequently misused. Cohen's Kappa has also been repeatedly criticized for the same reason (Allouche, Tsoar, \& Kadmon, 2006; Lobo et al., 2010; McPherson, Jetz, \& Rogers, 2004). TSS (Peirce, 1884), on the other hand, has fared relatively well since its introduction by Allouche et al. (2006) (719 citations in June 2017), mainly because it had been shown as independent of prevalence. However, this claim has recently been questioned because of a flawed testing design (Somodi, Lepesi, \& Botta-Dukát, 2017). More recently, all of these metrics have been contested because they should be calculated on presence-absence data, but are often used on presence-only or presence-background data, i.e. data with no information on locations where species do not occur (Jarnevich, Stohlgren, Kumar, Morisette, \& Holcombe, 2015; Somodi et al., 2017; Yackulic et al., 2013). In these cases, false positives and true negatives (Table 1) are unreliable, which led Li and Guo (2013) to propose alternative approaches, specifically designed for presence-background models. They proposed the use of $F_{\mathrm{pb}}$, a proxy of an F-measure ("the weighted harmonic average of precision and recall", Li \& Guo 2013) based on presence-background data, and $F_{\mathrm{cpb}}$, a prevalence-calibrated proxy of an $F$-measure based on presence-background data. Despite the apparent relevance of $\mathrm{Li}$ and Guo's (2013) metrics (13 citations as of June 2017), the field is stil dominated by metrics that have been repeatedly criticized, such as AUC and Kappa, or more recently TSS (e.g. D'Amen, Pradervand, \& Guisan, 2015; Jarnevich et al., 2015; Mainali et al., 2015).

With this Perspective, our aim is twofold: (a) illustrate with examples and simulations that, contrary to early claims, TSS is in fact dependent on prevalence and (b) evaluate an alternative set of metrics based on similarity indices, also known as F-measures in the binary classification literature, as potential alternative measures of model predictive ability. Similarity indices assess the similarity of observed and predicted distributions, and can be partitioned into two components to evaluate model characteristics: over prediction rate (OPR) and unpredicted presence rate (UPR). We compare the performance of TSS and similarity-derived metrics on three modelling situations corresponding to the most common modelling setups, depending on the interplay between species and sample prevalence (see Section 2). We finally discuss the applicability of

TABLE 1 Confusion matrix used to calculate discrimination metrics

\begin{tabular}{lll} 
& & Sampled data \\
\cline { 2 - 3 } & Presence & Absence \\
\hline Predicted values & & \\
Presence & True positives & False positives \\
\hline Absence & False negatives & True negatives \\
\hline
\end{tabular}

these discrimination metrics in SDM studies and provide practical recommendations.

\section{SPECIES AND SAMPLE PREVALENCE}

Here we will define species prevalence as the ratio between the species area of occupancy (AOO, i.e. the area that a species actually occupies) and the total study area (see Rondinini, Wilson, Boitani, Grantham, \& Possingham, 2006 for definitions). For example, if the study area encompasses Europe which we have divided into 1-km grid cells, and if we are studying a species that occupies only $15 \%$ of those grid cells, its prevalence would be 0.15 . Notice that species prevalence will vary depending on the resolution of the gridded data and on the extent of the study area. In practice, however, species prevalence is never known, because the true $\mathrm{AOO}$ is generally not known, except for the specific case of virtual species (Leroy et al., 2015). Hence, for real species, only the sample prevalence is known, which is the proportion of sampled sites in which the species has been recorded. Meynard and Kaplan (2012) showed with virtual species that sample prevalence should be similar to species prevalence to produce accurate predictions. However, in practice, we expect sample prevalence to be different from species prevalence, unless the sampling of presences and absences is perfectly random throughout the entire study area. Indeed, samplings of species presences are generally spatially biased (Phillips et al., 2009; Varela, Anderson, García-Valdés, \& FernándezGonzález, 2014). For example, ecologists look for their species of interest in sites where they have a sense a priori that they will find it, which will inevitably result in a mismatch between sample and species prevalence. Furthermore, a substantial proportion of SDM studies relies on presence-only modelling techniques, which requires sampling "pseudoabsence" or "background" points (hereafter called pseudoabsences). In such cases, the sample prevalence is artificially defined by the number of chosen pseudoabsences, and is thus unlikely to be equal to species prevalence.

Neither species prevalence nor sample prevalence should influence accuracy metrics. In the following, we investigate three different cases corresponding to the most common situations of SDM evaluation. First, we investigate the ideal "presence-absence" case where species prevalence is equal to sample prevalence; this case corresponds to well-designed presence-absence sampling or to the evaluation of SDMs based on virtual species where the true AOO is known. Second, we investigate "presence-absence" situations where sample prevalence differs from species prevalence. Last, we investigate "presence only" situations where sample prevalence differs from species prevalence.

\section{3 | PRESENCE-ABSENCE, SPECIES PREVALENCE $=$ SAMPLE PREVALENCE}

In this first case, we define the sample confusion matrix as perfectly proportional or equal to the true confusion matrix, i.e. the entire 
TABLE 2 Existing discrimination metrics

\begin{tabular}{lll} 
Metric & Calculation & References \\
\hline Sensitivity & $\mathrm{Sn}=\mathrm{TP} /(\mathrm{TP}+\mathrm{FN})$ & Fielding and Bell (1997) \\
\hline Specificity & $\mathrm{Sp}=\mathrm{TN} /(\mathrm{TN}+\mathrm{FP})$ & Fielding and Bell (1997) \\
\hline True skill statistic & $\mathrm{TSS}=\mathrm{Sn}+\mathrm{Sp}-1$ & Peirce (1884), Allouche et al. (2006) \\
\hline Jaccard's similarity index & Jaccard $=\mathrm{TP} /(\mathrm{FN}+\mathrm{TP}+\mathrm{FP})$ & Jaccard (1908) \\
\hline Sørensen's similarity index, F-measure & $\mathrm{S} \phi r e n s e n=2 \mathrm{TP} /(\mathrm{FN}+2 \mathrm{TP}+\mathrm{FP})$ & Sørensen (1948), Li and Guo (2013) \\
\hline $\begin{array}{l}\text { Proxy of F-measure } \\
\text { based on presence-background data }\end{array}$ & $\mathrm{F}_{\mathrm{pb}}=2 \times$ Jaccard & Li and Guo (2013) \\
\hline $\begin{array}{l}\text { Overprediction rate } \\
\text { Underprediction rate }\end{array}$ & $\mathrm{OPR}=\mathrm{FP} /(\mathrm{TP}+\mathrm{FP})$ & Barbosa et al. (2013) \\
\hline
\end{tabular}

TP: true positives, FN: false negatives, FP: false positives, TN: true negatives, P: number of sampled presences, $A$ : number of sampled pseudoabsences, prev $v_{\mathrm{sp}}$ : estimate of species prevalence.

predicted species distribution is compared to the true species distribution. In practice, this case occurs when the sampling is perfectly random throughout the landscape and species detectability is equal to one, or when evaluating SDM performance with virtual species (e.g. Qiao, Soberón, \& Peterson, 2015). With this first case we can analyse the sensitivity of discrimination metrics to species prevalence only.

\section{1 | The unexpected dependence of TSS on prevalence}

Previous studies have already shown that common discrimination metrics such as Kappa and AUC are influenced by species prevalence (e.g. Lobo et al., 2008, 2010). However, TSS has been widely advocated as a suitable discrimination metric that is independent of prevalence (Allouche et al., 2006). Here we demonstrate with simple examples that TSS is itself also dependent on species prevalence. When species prevalence is very low (and so is sample prevalence), we expect the number of true negatives (Table 1 ) to be disproportionately high. In these cases, specificity will tend towards one, and TSS values will be approximately equal to sensitivity (Table 2). As a result, TSS values can be high even for models that strongly overpredict distributions. Figure 1 represents graphically some examples of how overprediction and underprediction play into TSS performance. For example, Figure 1a shows a model that strongly overpredicts the distribution (75\% of the predicted distribution is composed of false positives), and yet TSS is close to 1 (Figure $1 \mathrm{a}$, TSS $=0.97$ ). Such a high value can in turn be produced by a model which correctly predicts the true distribution with few overpredictions (Figure 1b, TSS = 1.00). In addition, the overpredicting model (Figure 1a) will also have higher TSS values compared to a model that only missed 15\% of presences (Figure 1c, TSS $=0.85$ ). Furthermore, for identically performing models, if sample prevalence decreases (from 0.25 to 0.01 ), then the proportion of true negatives is increased, and consequently TSS values increased from 0.60 to 0.70 (Figure $1 \mathrm{~d}-\mathrm{f}$ ). Consequently, TSS values can be artificially increased by decreasing sample prevalence. As an unexpected consequence, for two species with different AOO in the study area (thus different sample prevalence), the species with the smaller distribution will be considered better predicted than the one with a larger distribution (Figure 1d-f).

To summarize, TSS values can be misleading in situations where the number of true negatives is high by (a) not penalizing overprediction and (b) assigning higher values to models of species with lower prevalence in cases of two models with identical discrimination accuracy. These flaws can be strongly problematic for ecologists, and during SDM performance evaluation it is generally preferable to assume that overprediction should be equivalent to underprediction (e.g. Lawson, Hodgson, Wilson, \& Richards, 2014). Therefore, we conclude that TSS is prone to similar shortcomings as AUC and Kappa when it comes to its dependence on sample prevalence and AOO.

\subsection{Similarity metrics as an alternative}

To avoid these shortcomings, we propose to focus evaluation metrics on three components of the confusion matrix (Table 1): true positives, false positives and false negatives, neglecting the true negatives that could be easily inflated. In particular, we seek to maximize true positives, and minimize both false positives and false negatives with respect to true positives. The definition exactly matches the definition of similarity indices from community ecology, such as Jaccard and Sørensen indices or the F-measure indices (Table 2). This definition also matches indices identified by $\mathrm{Li}$ and Guo (2013) as potential presence-background metrics. The $F_{p b}$ index is in fact equal to twice the Jaccard index (eqn. 13 in Li \& Guo, 2013), while the $F$ index is equal to the Sørensen index of similarity (eqn. 4 in Li \& Guo, 2013; Table 2).

Similarity indices have two main benefits. First, their conceptual basis is easy to understand: they measure the similarity between predictions and observations. A value of 1 means predictions perfectly match observations, without any false positive or false negative. A value of 0 means that none of the predictions matched any observation. The lower the similarity value, the higher the number of false positives and false negatives, relative to the number of true 


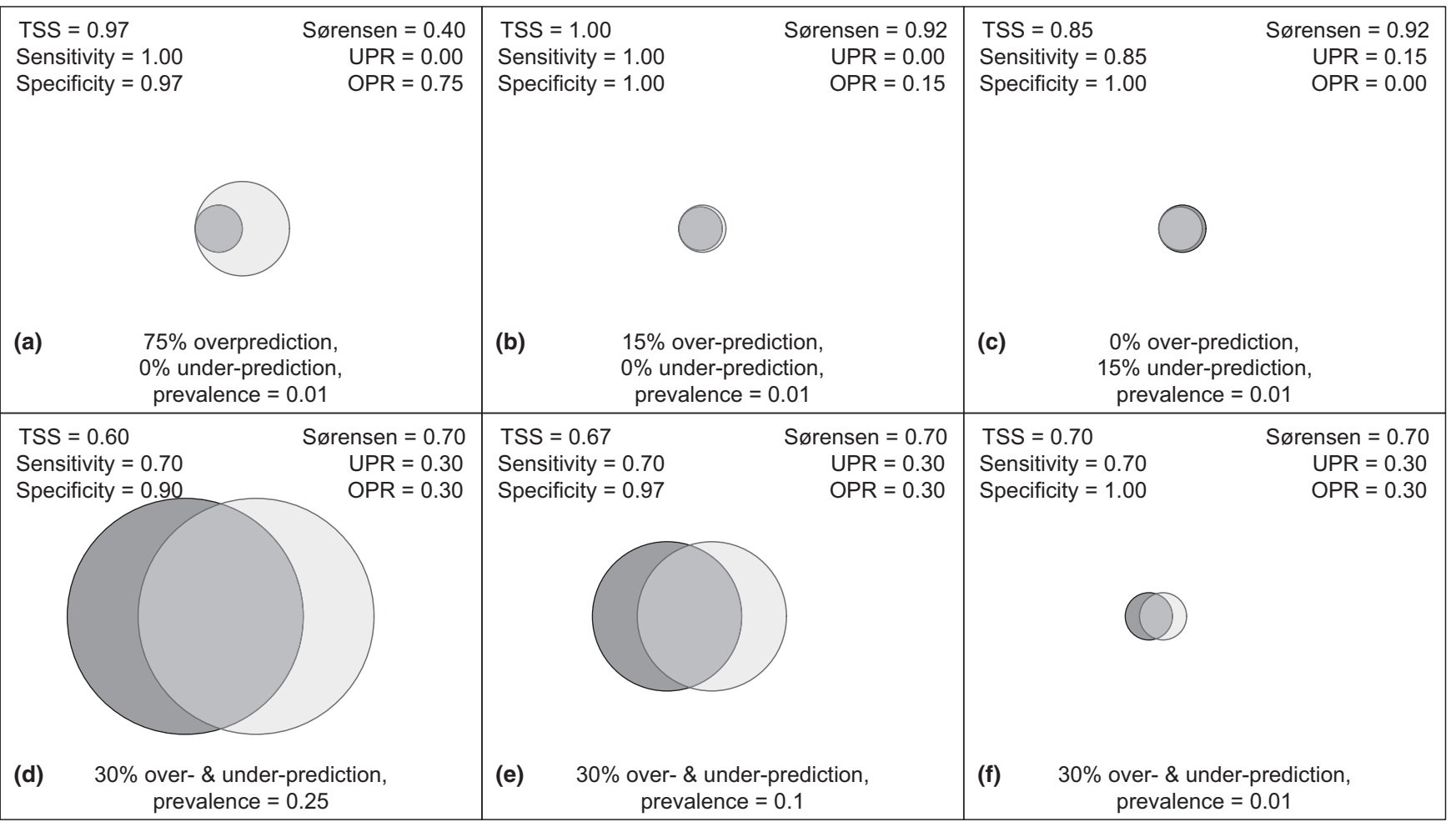

FIGURE 1 Examples of model performances and associated metrics. The dark grey-filled circle represents the proportion of actual presences in the sample. The light grey-filled circle represents the proportion of predicted presences in the sample. Therefore, the overlap between the two circles represents the proportion of actual presences correctly predicted as presences ("true positives"), whereas the white area represents the proportion of actual absences correctly predicted as absences ("true negatives"). At low prevalence (0.10), TSS does not penalize overprediction: a model that strongly overpredicts distribution (a; 75\% of the predicted distribution is composed of false positives) can have a very high TSS (0.97), which is almost equivalent to a model with little overprediction $(b$, TSS $=1.00)$. TSS does penalize underprediction $(c$, TSS $=0.85$ ) much more than overprediction $(a, b)$. For identically performing models (i.e. similar rates of over- and underprediction), if prevalence decreases (from 0.25 to 0.01) with increasing numbers of true negatives, TSS values increased from 0.60 to 0.70 (d-f). In other words, for two species with different AOO in a given study area, the species with the smaller distribution has a higher TSS than the one with a larger distribution. Sørensen, on the other hand, accurately discriminates between highly overpredicting and well performing models (a-c). Similarity indices penalize identically over- and underprediction (b,c). In addition, when species prevalence is artificially increased for identical models, both indices remain identical $(\mathrm{d}-\mathrm{f})$

presences. Second, as similarity indices do not include true negatives, they are not biased by a disproportionate number of true negatives. In return, they do not estimate the capacity of models to correctly predict absences. To illustrate this, we calculated the Sørensen index of similarity ( $F$-measure) on the same examples as above. Sørensen accurately discriminated between highly overpredicting and well performing models (Figure 1a-c). In addition, when species prevalence was artificially increased for identical models, both indices remained identical (Figure 1d-f).

Because the specific objectives of SDM studies can be very different (e.g. invasion monitoring versus habitat identification for threatened species), in a particular context we may be more interested in assessing whether predictions tend to over- or underestimate observations. Such additional information can be obtained with metrics derived from the confusion matrix: overprediction rate and UPR (Table 2). The overprediction rate measures the percentage of predicted presences corresponding to false presences, and was already recommended for assessing model overprediction (Barbosa,
Real, Muñoz, \& Brown, 2013). The UPR measures the percentage of actual presences not predicted by the model, and is also called the false-negative rate (Fielding \& Bell, 1997). Taken together these metrics provide a full view of model discrimination accuracy and allow interpreting the results in the specific context of the study.

\section{3 | Demonstration based on simulations}

To validate these theoretical demonstrations, we performed simulations of the metrics for three case studies with different performances: a first model with $40 \%$ overprediction and $40 \%$ underprediction, a second model with $40 \%$ underprediction and no overprediction, and a third model with $40 \%$ overprediction and no underprediction. The first case addresses a predicted range that is shifted in space with respect to the real one; the second and third cases address situations where the predicted range is, respectively, smaller or larger than the real one. For each model, we predicted the distribution range of theoretical species with different prevalences 
(from 0.01 to 0.60 with a step of 0.01 ) over an area of 100,000 pixels. Then, for each species, we randomly sampled 500 presences in the total area and a number of absences verifying the condition that the sample prevalence is equal to species prevalence. We repeated this procedure five times. For each repetition, we calculated the TSS and the Sørensen index (R scripts available at https://github.com/Fa rewe/SDMMetrics).

Our results (Figure 2) show that TSS values decreased with prevalence for cases that overpredicted species distributions, but not for cases that only underpredicted distributions (Figure 2a). This result confirms our expectation that TSS does not penalize overprediction at low prevalence. Sørensen values, on the other hand, remain similar regardless of species prevalence (Figure $2 b$ ). These results confirm that in the ideal situation where species prevalence $=$ sample prevalence, the Sørensen index of similarity is a more appropriate metric of model discrimination capacity.

\section{PRESENCE-ABSENCE, SPECIES PREVALENCE $\neq$ SAMPLE PREVALENCE}

When sample prevalence is different from species prevalence, the ratio of sampled absences over sampled presences is different from the ratio of true absences over true presences. For example, if too many absences are sampled (sample prevalence lower than species prevalence), then the numbers of false positives and true negatives will be too large compared to true positives and false negatives. The major consequence of this mismatch is that any metric comparing sampled presences and absences will not reflect true model performance, unless it contains a correction factor for the mismatch between sample and species prevalence. Note, however, that metrics focusing only on sampled presences (omitting sampled absences) will not be affected by this bias (e.g. sensitivity or rate of unpredicted presences will not be affected). We illustrate in Supporting

\section{Case studies $40 \%$ overprediction \& $40 \%$ underprediction $40 \%$ underprediction $40 \%$ overprediction}

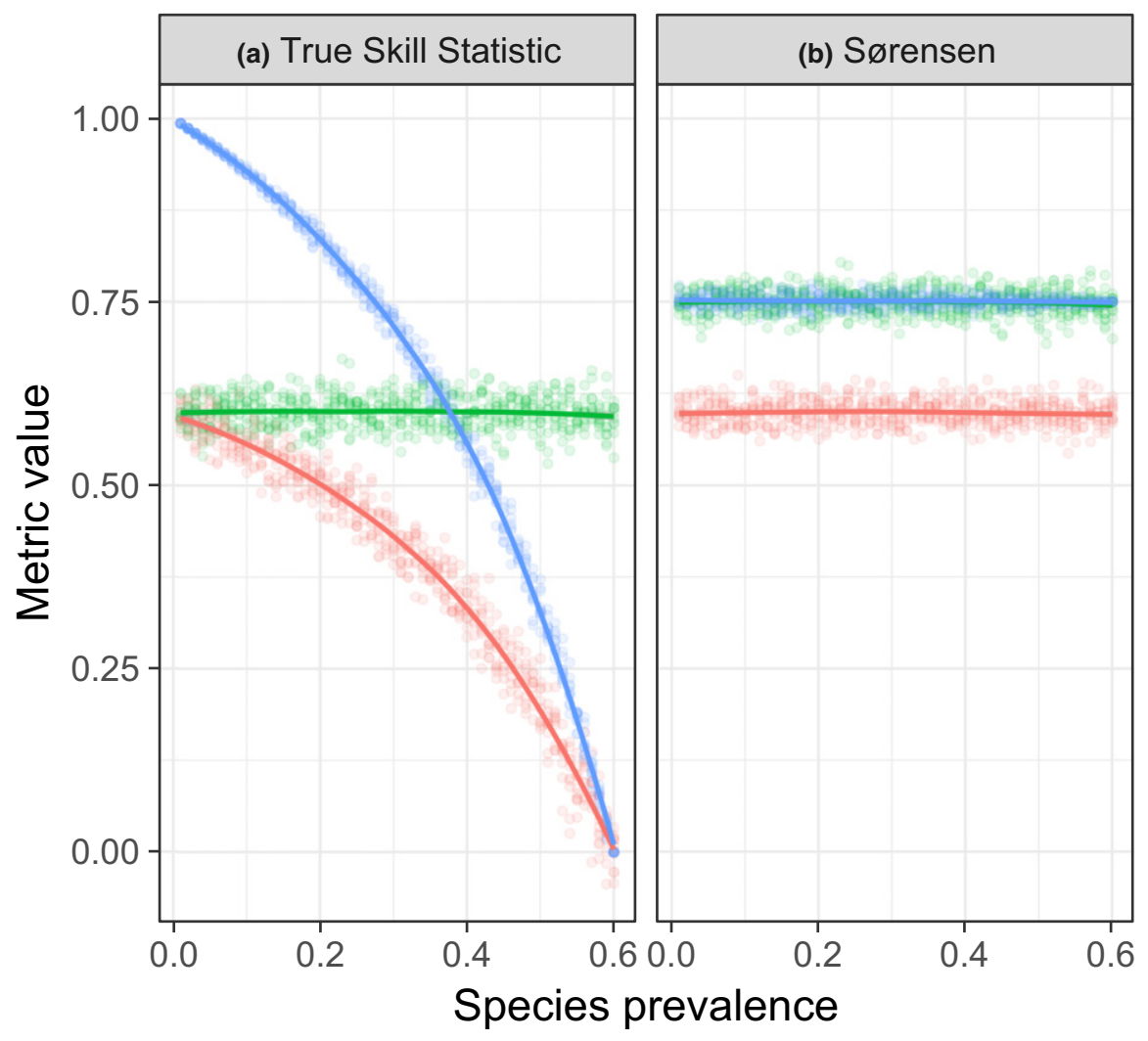

FIGURE 2 Simulations of the effect of species prevalence on species distribution model discrimination metrics (a) TSS and (b) Sørensen (equations available in Table 2) in a presence-absence scheme where sample prevalence is equal to species prevalence. Three case studies with varying degrees of over- and underprediction are applied to theoretical species with prevalence ranging from 0.01 to 0.60 with a step of 0.01 . The upper limit of 0.60 was chosen to produce values for models with $40 \%$ overprediction. For each species, an evaluation dataset was composed of 500 presences randomly sampled in the total area and a number of randomly sampled absences verifying the condition that the sample prevalence is equal to species prevalence, with five repetitions for each species (R scripts available at https://github.com/Farewe/ SDMMetrics). These simulations showed that TSS attributes higher values at lower prevalence for case studies that overpredict species distributions, but not for case studies that have only underprediction (a). Sørensen values, on the other hand, remain similar regardless of species prevalence (b) 
Information Appendix S1 how the aforementioned metrics are biased by prevalence in this situation: the lower the prevalence, the higher the metric. We further show that an appropriate estimation can only be obtained when an accurate estimation of species prevalence is available, which is generally not the case (see Section 6).

\section{5 | PRESENCE-PSEUDOABSENCE OR PRESENCE-BACKGROUND, SPECIES PREVALENCE $\neq$ SAMPLE PREVALENCE}

In presence-pseudoabsence schemes, sample prevalence is highly unlikely to be equal to species prevalence, thus the previous bias also applies in this situation. Furthermore, an additional bias is added by the fact that pseudoabsence points may be actual presence points. This bias will further impact the estimation of false positive by generating "false false positives", i.e. predicted presences corresponding to actual presences but sampled as pseudoabsences. We illustrate with simulation how this bias increases the dependence on prevalence of existing metrics in Supporting Information Appendix S2, including the prevalence-calibrated $F_{\mathrm{cpb}}$ metric specifically designed for presence-background (Li \& Guo, 2013). We also illustrate that a mathematical correction could be applied but requires ideal conditions unlikely to be obtained (perfectly random samplings of presences and pseudoabsences, multiple repetitions, accurate estimation of species prevalence; see section Estimations of species prevalence).

\section{6 | ESTIMATIONS OF SPECIES PREVALENCE}

The only way to correct discrimination metrics in cases where sample prevalence is different from species prevalence requires an estimate of species prevalence. In presence-absence schemes, species prevalence is usually estimated from the sample of presences and absences-however we assumed here that in many situations this estimate may be biased. Besides, in presence-pseudoabsence schemes this estimation is not available. An alternative approach consists in estimating species prevalence from the modelled species distribution (e.g. Li \& Guo, 2013; Liu, Newell, \& White, 2016). Li and Guo (2013) demonstrated that this approach yielded satisfactory results for presence-pseudoabsence based on the $F_{\mathrm{pb}}$ index. However, these results were later contested by Liu et al. (2016) who found that neither $F_{\mathrm{pb}}$, nor a TSS-derived metric were able to correctly estimate species prevalence with presence-pseudoabsence data. This inability to estimate species prevalence from presencepseudoabsence data was expected because an accurate estimation would require strong conditions which are unlikely to be met in reality (see Guillera-Arroita et al., 2015 for a demonstration). Actually, for both presence-pseudoabsence and presence-absence data, estimating species prevalence could be feasible from limited presenceabsence surveys, but may be prohibitively difficult or expensive to obtain (Lawson et al., 2014; Phillips \& Elith, 2013). This barrier to estimating species prevalence severely limits the applicability of discrimination metrics for presence-absence and presence-pseudoabsence models where sample prevalence is different from species prevalence.

\section{7 | DISCUSSION AND RECOMMENDATIONS}

In this paper, we have demonstrated that evaluating model discrimination capacity (i.e. the capacity to accurately discriminate between presence and absence) depends on the interplay between sample and species prevalence. We studied three general situations that modellers frequently encounter in their modelling exercises: (a) a presence-absence scheme where sample prevalence is equal to species prevalence-this situation corresponds to perfectly random presence-absence samplings with no detection bias, or to evaluations based on virtual species; (b) a presence-absence scheme where sample prevalence is different from species prevalence-a likely situation for presence-absence modelling; and (c) a presence-pseudoabsence scheme where sample prevalence is different from species prevalence-the general case for presence-pseudoabsence or presence-background modelling.

Our simulations unequivocally indicate that when sample prevalence is different from species prevalence, none of the tested metrics are independent of species prevalence, corroborating previous conclusions on the TSS (Somodi et al., 2017), and invalidating the propositions of $\mathrm{Li}$ and Guo (2013). Our rationale and conclusions on TSS relate in fact to the same argumentation as provided on AUC by Lobo et al. (2008). Both TSS and AUC have the same shortcomings. Most importantly, Lobo et al. (2008) showed that the total spatial extent used to calibrate a species' model highly influenced AUC values. Indeed, the total study extent drives species prevalence (termed Relative Occurrence Area in Lobo et al., 2008); increasing extent reduces species prevalence and vice versa. Consequently, artificially increasing the modelling extent will artificially decrease prevalence, which in turn will increase AUC values (Jiménez-Valverde, Acevedo, Barbosa, Lobo, \& Real, 2013; Lobo et al., 2010), but also TSS values as we showed here. Likewise, comparing species with different AOO over the same extent will provide an unfair advantage to species with smaller AOO because they will have a smaller prevalence. In fact, these shortcomings are likely to extend to any measurement that need to estimate either false positive or true negative (JiménezValverde et al., 2013).

Our first recommendation is a compelling advocacy for improving data quality in SDMs. Our arguments, as well as those of Lobo et al. (2008, 2010) and Jiménez-Valverde et al. (2013), suggest that the quest for an ideal discrimination metric is futile, unless reliable presence-absence data are available. Indeed, an unbiased set of presence and absence data is required to estimate species prevalence (Guillera-Arroita et al., 2015), and any metric based on true negative and false positive (Jiménez-Valverde et al., 2013). Therefore, we advocate 
the importance of collecting more informative data. Ideally, we emphasize the necessity of obtaining at least a random or representative sample of presences and absences (Phillips \& Elith, 2013), or of improving data collection, for instance, by recording non-detections to estimate sampling bias and species prevalence (GuilleraArroita et al., 2015; Lahoz-Monfort, Guillera-Arroita, \& Wintle, 2014). Cross-validation procedures can lead to overoptimistic evaluations because of data autocorrelation, and specific procedures can be applied to avoid this further bias (Roberts et al., 2017). We also emphasize the importance of appropriate spatial extent; although a robust framework for choosing spatial extent does not exist, guidelines exist to improve spatial extent definition (Barve et al., 2011; Jarnevich et al., 2015).

Our second recommendation concerns the case where quality presence-absence data are available. This is also the case of virtual species, which are increasingly used to develop and test SDM methodologies (Hattab et al., 2017; Leroy et al., 2015; Li \& Guo, 2013; Liu et al., 2016; Meynard \& Kaplan, 2013; Miller, 2014; Ranc et al., 2016; Varela et al., 2014). Our results unequivocally demonstrated that similarity/F-measure metrics, and their complementary metrics (OPR, UPR) were unbiased by species prevalence and, thus, can be applied to produce discrimination metrics with better performance than Kappa, AUC and TSS metrics. Therefore, we strongly recommend the use of similarity/F-measures in the specific case of virtual species. After all, virtual species are used to demonstrate the shortcoming and/or advantages of some methods over others, and therefore the use of appropriate evaluation metrics is highly desirable.

\section{ACKNOWLEDGEMENTS}

We sincerely thank Jorge $M$. Lobo and two anonymous reviewers for their significant contribution in the improvement of this manuscript.

\section{Keywords}

AUC, ecological niche models, model evaluation, prevalence, species distribution models

\section{ORCID}

Boris Leroy (iD http://orcid.org/0000-0002-7686-4302

Christine N. Meynard iD http://orcid.org/0000-0002-5983-6289

Boris Leroy ${ }^{1}$

Robin Delsol ${ }^{1,2}$

Bernard Hugueny ${ }^{3}$

Christine N. Meynard ${ }^{4}$ iD

Chéima Barhoumi ${ }^{1,2,5}$

Morgane Barbet-Massin ${ }^{2}$

Céline Bellard ${ }^{1,6}$
${ }^{1}$ Unité Biologie des Organismes et Ecosystèmes Aquatiques (BOREA UMR 7208), Muséum National d'Histoire Naturelle, Sorbonne Universités, Université de Caen Normandie, Université des Antilles, CNRS, IRD, Paris, France

${ }^{2}$ Ecologie, Systématique \& Evolution, UMR CNRS 8079, Univ. Paris-Sud,

Orsay Cedex, France

${ }^{3}$ Laboratoire Évolution \& Diversité Biologique (EDB UMR 5174), Université de Toulouse Midi-Pyrénées, CNRS, IRD, UPS, Toulouse Cedex

${ }^{4}$ CBGP, INRA, CIRAD, IRD, Montpellier SupAgro, Univ Montpellier, Montpellier, France

${ }^{5}$ Institut des Sciences de l'Evolution de Montpellier, UMR CNRS 5554, Univ. De Montpellier, Montpellier Cedex, France

${ }^{6}$ Department of Genetics, Evolution and Environment, Center for Biodiversity and Environment Research, University College of London, London, UK

Correspondence

Boris Leroy, Unité Biologie des Organismes et Ecosystèmes Aquatiques (BOREA, UMR 7208), Muséum National d'Histoire Naturelle, Sorbonne Universités, Université de Caen Normandie, Université des Antilles, CNRS, IRD, Paris, France.

Email: leroy.boris@gmail.com

\section{REFERENCES}

Allouche, O., Tsoar, A., \& Kadmon, R. (2006). Assessing the accuracy of species distribution models: Prevalence, kappa and the true skill statistic (TSS). Journal of Applied Ecology, 43(6), 1223-1232. https://d oi.org/10.1111/j.1365-2664.2006.01214.x

Araújo, M. B., Pearson, R. G., Thuiller, W., \& Erhard, M. (2005). Validation of species-climate impact models under climate change. Global Change Biology, 11(9), 1504-1513. https://doi.org/10.1111/j.13652486.2005.01000.x

Barbosa, A. M., Real, R., Muñoz, A. R., \& Brown, J. A. (2013). New measures for assessing model equilibrium and prediction mismatch in species distribution models. Diversity and Distributions, 19(10), 13331338. https://doi.org/10.1111/ddi.12100

Barve, N., Barve, V., Jiménez-Valverde, A., Lira-Noriega, A., Maher, S. P., Peterson, A. T., ... Villalobos, F. (2011). The crucial role of the accessible area in ecological niche modeling and species distribution modeling. Ecological Modelling, 222(11), 1810-1819. https://doi.org/10. 1016/j.ecolmodel.2011.02.011

Bellard, C., Thuiller, W., Leroy, B., Genovesi, P., Bakkenes, M., \& Courchamp, F. (2013). Will climate change promote future invasions? Global Change Biology, 19(12), 3740-3748. https://doi.org/10.1111/gcb. 12344

Boyce, M. S., Vernier, P. R., Nielsen, S. E., \& Schmiegelow, F. K. (2002). Evaluating resource selection functions. Ecological Modelling, 157 (2-3), 281-300. https://doi.org/10.1016/S0304-3800(02)00200-4

D'Amen, M., Pradervand, J.-N., \& Guisan, A. (2015). Predicting richness and composition in mountain insect communities at high resolution: A new test of the SESAM framework. Global Ecology and Biogeography, 24, 1443-1453. https://doi.org/10.1111/geb.12357

Di Cola, V., Broennimann, O., Petitpierre, B., Breiner, F. T., D'Amen, M., Randin, C, ... Guisan, A. (2016). ecospat: An R package to support spatial analyses and modeling of species niches and distributions. Ecography, 40, 774-787.

Fielding, A. H., \& Bell, J. F. (1997). A review of methods for the assessment of prediction errors in conservation presence/absence models. 
Environmental Conservation, 24(1), 38-49. https://doi.org/10.1017/ S0376892997000088

Gallon, R. K., Robuchon, M., Leroy, B., Le Gall, L., Valero, M., \& Feunteun, E. (2014). Twenty years of observed and predicted changes in subtidal red seaweed assemblages along a biogeographical transition zone: Inferring potential causes from environmental data. Journal of Biogeography, 41(12), 2293-2306. https://doi.org/10.1111/jbi.12380

Guillera-Arroita, G., Lahoz-Monfort, J. J., Elith, J., Gordon, A., Kujala, H., Lentini, P. E., ... Wintle, B. A. (2015). Is my species distribution model fit for purpose? Matching data and models to applications. Global Ecology and Biogeography, 24(3), 276-292. https://doi.org/10.1111/ geb.12268

Guisan, A., Tingley, R., Baumgartner, J. B., Naujokaitis-Lewis, I., Sutcliffe, P. R., Tulloch, A. I. T., ... Buckley, Y. M. (2013). Predicting species distributions for conservation decisions. Ecology Letters, 16(12), 1424 1435. https://doi.org/10.1111/ele.12189

Hattab, T., Garzón-López, C. X., Ewald, M., Skowronek, S., Aerts, R., Horen, H, ... Lenoir, J. (2017). A unified framework to model the potential and realized distributions of invasive species within the invaded range. Diversity and Distributions, 23, 1-14.

Hirzel, A. H., Randin, C., \& Guisan, A. (2006). Evaluating the ability of habitat suitability models to predict species presences. Ecological Modelling, 199, 142-152. https://doi.org/10.1016/j.ecolmodel.2006. 05.017

Jaccard, P. (1908). Nouvelles recherches sur la distribution florale. Bulletin de la Societe Vaudoise des Sciences Naturelles, 44, 223-270.

Jarnevich, C. S., Stohlgren, T. J., Kumar, S., Morisette, J. T., \& Holcombe, T. R. (2015). Caveats for correlative species distribution modeling. Ecological Informatics, 29, 6-15. https://doi.org/10.1016/j.ecoinf. 2015.06.007

Jiménez-Valverde, A. (2012). Insights into the area under the receiver operating characteristic curve (AUC) as a discrimination measure in species distribution modelling. Global Ecology and Biogeography, 21(4), 498-507. https://doi.org/10.1111/j.1466-8238.2011.00683.x

Jiménez-Valverde, A., Acevedo, P., Barbosa, A. M., Lobo, J. M., \& Real, R. (2013). Discrimination capacity in species distribution models depends on the representativeness of the environmental domain. Global Ecology and Biogeography, 22(4), 508-516. https://doi.org/10. 1111/geb.12007

Lahoz-Monfort, J. J., Guillera-Arroita, G., \& Wintle, B. A. (2014). Imperfect detection impacts the performance of species distribution models. Global Ecology and Biogeography, 23(4), 504-515. https://doi.org/ 10.1111/geb.12138

Lawson, C. R., Hodgson, J. A., Wilson, R. J., \& Richards, S. A. (2014). Prevalence, thresholds and the performance of presence-absence models. Methods in Ecology and Evolution, 5(1), 54-64. https://doi. org/10.1111/2041-210X.12123

Leroy, B., Bellard, C., Dubos, N., Colliot, A., Vasseur, M., Courtial, C., ... Ysnel, F. (2014). Forecasted climate and land use changes, and protected areas: The contrasting case of spiders. Diversity and Distributions, 20, 686-697. https://doi.org/10.1111/ddi.12191

Leroy, B., Meynard, C. N., Bellard, C., \& Courchamp, F. (2015). virtualspecies, an $\mathrm{R}$ package to generate virtual species distributions. Ecography, 39, 599-607.

Li, W., \& Guo, Q. (2013). How to assess the prediction accuracy of species presence-absence models without absence data? Ecography, 36 (7), 788-799. https://doi.org/10.1111/j.1600-0587.2013.07585.x

Liu, C., Newell, G., \& White, M. (2016). On the selection of thresholds for predicting species occurrence with presence-only data. Ecology and Evolution, 6(1), 337-348. https://doi.org/10.1002/ece3.1878

Liu, C., White, M., \& Newell, G. (2009). Measuring the accuracy of species distribution models: A review. 18th World IMACS/MODSIM Congress, Cairns, Australia 13-17 July, 4241-4247.

Lobo, J. M., Jiménez-Valverde, A., \& Hortal, J. (2010). The uncertain nature of absences and their importance in species distribution modelling. Ecography, 33(1), 103-114. https://doi.org/10.1111/j. 1600-0587.2009.06039.x

Lobo, J. M., Jiménez-Valverde, A., \& Real, R. (2008). AUC: A misleading measure of the performance of predictive distribution models. Global Ecology and Biogeography, 17(2), 145-151. https://doi.org/10.1111/j. 1466-8238.2007.00358.x

Mainali, K. P., Warren, D. L., Dhileepan, K., McConnachie, A., Strathie, L., Hassan, G., ... Parmesan, C. (2015). Projecting future expansion of invasive species: Comparing and improving methodologies for species distribution modeling. Global Change Biology, 21(12), 4464-4480. https://doi.org/10.1111/gcb.13038

McPherson, J. M., Jetz, W., \& Rogers, D. J. (2004). The effects of species' range sizes on the accuracy of distribution models: Ecological phenomenon or statistical artefact? Journal of Applied Ecology, 41(5), 811-823. https://doi.org/10.1111/j.0021-8901.2004.00943.x

Meynard, C. N., \& Kaplan, D. M. (2012). The effect of a gradual response to the environment on species distribution modeling performance. Ecography, 35(6), 499-509. https://doi.org/10.1111/j.1600-0587. 2011.07157.x

Meynard, C. N., \& Kaplan, D. M. (2013). Using virtual species to study species distributions and model performance. Journal of Biogeography, 40(1), 1-8. https://doi.org/10.1111/jbi.12006

Miller, J. A. (2014). Virtual species distribution models: Using simulated data to evaluate aspects of model performance. Progress in Physical Geography, 38(1), 117-128. https://doi.org/10.1177/ 0309133314521448

Pearce, J., Pearce, J., Ferrier, S., \& Ferrier, S. (2000). Evaluating the predictive performance of habitat models developed using logistic regression. Ecological Modelling, 133, 225-245. https://doi.org/10. 1016/S0304-3800(00)00322-7

Peirce, C. S. (1884). The numerical measure of the success of prediction. Science, 4(93), 453-454. https://doi.org/10.1126/science.ns-4.93.453-a

Phillips, S. J., Dudík, M., Elith, J., Graham, C. H., Lehmann, A., Leathwick, J., \& Ferrier, S. (2009). Sample selection bias and presence-only distribution models: Implications for background and pseudo-absence data. Ecological Applications, 19(1), 181-197. https://doi.org/10.1890/072153.1

Phillips, S. J., \& Elith, J. (2013). On estimating probability of presence from use-availability or presence-background data. Ecology, 94(6), 1409-1419. https://doi.org/10.1890/12-1520.1

Qiao, H., Soberón, J., \& Peterson, A. T. (2015). No silver bullets in correlative ecological niche modeling: Insights from testing among many potential algorithms for Niche estimation. Methods in Ecology and Evolution, 6(10), 1126-1136. https://doi.org/10.1111/2041-210X.12397

Ranc, N., Santini, L., Rondinini, C., Boitani, L., Poitevin, F., Angerbjörn, A., \& Maiorano, L. (2016). Performance tradeoffs in target-group bias correction for species distribution models. Ecography, 40, 1-12.

Roberts, D. R., Bahn, V., Ciuti, S., Boyce, M. S., Elith, J., Guillera-Arroita, G., ... Dormann, C. F. (2017). Cross-validation strategies for data with temporal, spatial, hierarchical, or phylogenetic structure. Ecography, 40, 913-929. https://doi.org/10.1111/ecog.02881

Rondinini, C., Wilson, K. A., Boitani, L., Grantham, H., \& Possingham, H. P. (2006). Tradeoffs of different types of species occurrence data for use in systematic conservation planning. Ecology Letters, 9(10), 11361145. https://doi.org/10.1111/j.1461-0248.2006.00970.x

Somodi, I., Lepesi, N., \& Botta-Dukát, Z. (2017). Prevalence dependence in model goodness measures with special emphasis on true skill statistics. Ecology and Evolution, 7, 863-872. https://doi.org/10.1002/ ece 3.2654

Sørensen, T. (1948). A method of establishing groups of equal amplitude in plant sociology based on similarity of species and its application to analyses of the vegetation on Danish commons. Biologiske Skrifter/ Kongelige Danske Videnskabernes Selskab, 1-34.

Varela, S., Anderson, R. P., García-Valdés, R., \& Fernández-González, F. (2014). Environmental filters reduce the effects of sampling bias and 
improve predictions of ecological niche models. Ecography, 37, 1084 1091.

Yackulic, C. B., Chandler, R., Zipkin, E. F., Royle, J. A., Nichols, J. D., Campbell Grant, E. H., \& Veran, S. (2013). Presence-only modelling using MAXENT: When can we trust the inferences? Methods in Ecology and Evolution, 4(3), 236-243. https://doi.org/10.1111/2041-210x.12004

\section{BIOSKETCH}

Boris Leroy is lecturer at the Muséum National d'Histoire Naturelle of Paris. He is interested in the biogeography of aquatic organisms and how it is or will be impacted by global changes such as climate change and biological invasions.

\section{SUPPORTING INFORMATION}

Additional supporting information may be found online in the Supporting Information section at the end of the article.

How to cite this article: Leroy B, Delsol R, Hugueny B, et al. Without quality presence-absence data, discrimination metrics such as TSS can be misleading measures of model performance. J Biogeogr. 2018;00:1-9. https://doi.org/ 10.1111/jbi.13402 